\title{
THE EFFECT OF DIFFERENT PLANTING SYSTEMS ON THE GROWTH, YIELD AND FIBER QUALITY OF COTTON CULTIVARS
}

\author{
ALI, H. - HASSAN, W. - IRFAN, M. \\ Department of Agronomy, Faculty of Agricultural Sciences and Technology, Bahauddin \\ Zakariya University, Multan, Pakistan \\ *Corresponding author \\ e-mail:muhammad.irfan26@gmail.com \\ (Received $24^{\text {th }}$ Jun 2020; accepted $6^{\text {th }}$ Oct 2020)
}

\begin{abstract}
Tillage system is a critical factor that limits plant growth and production all over the globe. The current study was to evaluate the performance of cotton cultivars and appropriate planting system for higher crop production in Punjab, Pakistan. Two factors; cultivars and planting system were examined under RCBD along four repeats. "CIM-602 cultivar" had the highest yield traits i.e. plant height, number of bolls/ plant, boll weight, seed cotton yield, lint yield, biological yield and seed cotton harvest. The excellent increase was shown in yield and phenological attributes of cotton cultivars under bed sowing compared to all other studied planting systems. Regarding the cultivars, the higher fiber related attributes such as fiber fineness, fiber uniformity, fiber elongation, fiber strength and micronaire were measured in "CIM-602 cultivar" as compared to "CIM-678cultivar". The bed sowing provides the best fiber quality as compared to other planting systems. Economic analysis confirmed that bed sowing is best planting system which provides higher output to farmers with little input. It has been concluded that from present study, "CIM-602" is good cultivar and provides higher production, while bed sowing is suitable planting system for cotton production.
\end{abstract}

Keywords: fiber quality traits, economic analysis, growth related traits, physiological traits

\section{Introduction}

Cotton (Gossypium hirsutum L.) is famous as white gold and belongs to family Malvaceae. Three different species including Gossypium hirsutum, Gossipium arboretum as well as Gossypium barbedense are economically most important to fulfill fiber requirements worldwide (Zohaib et al., 2018). Among different countries, China is famous as one of the leading country for the cotton production worldwide because of its modern research and development (Pettigrew and Dowd, 2011). However, Pakistan ranks $4^{\text {th }}$ after China, USA and India regarding the cotton production than other countries (Amouzou et al., 2018). Cotton had excellent role in economy of Pakistan. Cotton is major oil producing crop of Pakistan. Therefore, cotton is acknowledging as major cash as well as fiber crop in Pakistan (Shah et al., 2011).

In Pakistan cotton production is very poor because of different biotic and abiotic stresses as compared to other developed countries. Hence numerous other factors i.e. insect pest and disease attack, selection of genotypes, different planting system, inadequate fertilizer application, nutritional imbalance and improper agronomic practices resulted in poor yield of cotton. The performance of cultivars can vary from cultivar to other because of its different genetic background (Ahmad et al., 2019). Cultivation of those genotypes having unique genetic background suitable for higher production is major requirement with passage of time (Bellaloui et al., 2015). Therefore, huge efforts 
were recommended for attaining higher yield regarding different low yielding aspects of cotton (Magare et al., 2018).

Though, massive efforts are requisite to improve crop growth which ultimately increase in cotton yield as well as improve cotton fiber quality. However, cotton cultivation is more in Pakistan than other agronomic crops but yield per hectare is found to be minimum because of different environmental constrains such as drought, salinity and many others (Zohaib et al., 2018). The shortage of water is big problem in Pakistan. Moreover, drought environments greatly reduced the plant growth and yield traits of many cotton cultivars. Therefore, numerous management practices such as cultivars development with superior traits, selection of higher yielding cultivars, cultivars potential against adverse environmental conditions, good fiber quality are needed for attaining higher production from (Pettigrew and Dowd, 2011).

Planting system is major constrain that is involved in production of different agronomic crops. Different yield reduction aspects were studied in previous work (Ali et al., 2010). However, examination of planting system is very negligible regarding cotton production. Suitable use of planting system is very important aspect for attaining higher production of cotton. Three different planting systems i.e. raised beds, flat beds and ridges are used for cotton cultivation (Nadeem et al., 2013). The most frequently used planting system is ridges cultivation since earlier times. However, many farmers more satisfied on ridges cultivation than other planting system. The excellent plant growth as well as production was recorded from ridges cultivation in numerous other crops (Anjum et al., 2019). In recent times, it has been identified that raised beds are greatly preferable than ridges under drought stress conditions because raised beds have excellent water holding potential than all other planting systems (Ali et al., 2015). Earlier research work indicated that planting system is successful involved in higher production of cotton. Huge research work is conducted on water stress conditions, while determination of suitable planting system under water stress conditions is novelty of the current work. Therefore, current study encourages the cultivation of drought resistant cultivars with suitable tillage system that will increase the cotton production.

\section{Materials and methods}

Present study was accomplished at Central Cotton Research Institute (CCRI) Multan in $2017 \& 2018$. Current site lies at a latitude of $30^{\circ}$, and longitude of $71^{\circ}$ and the altitude is 125 in the Punjab, Pakistan. Performance of two cultivars CIM-678 as well as CIM-602 was checked under three different planting systems such as flat, ridge and bed sowing. Experimental site was deeply ploughed and well prepared, become suitable for cotton cultivation. Experimental soil possessed organic matter $(0.86 \%)$, nitrogen $(0.09 \%)$ as well as phosphorous $(12.5 \mathrm{ppm})$. The experimental plot size was nearly $6.1 \mathrm{~m} \times 9.0 \mathrm{~m}$ with seed rate $110 \mathrm{~g} /$ plot. Crop was harvested from 07-11-2017 as well as 16-11-2018 with several pickings after 15 days interval.

Appropriate management practices i.e. timely irrigation, fertilizer application, weeds eradication and control of various insect pest and diseases were applied till crop harvesting. Urea fertilization was nearly $35 \mathrm{~kg} \mathrm{ha}^{-1}$. Irrigation was applied after one week interval as when it required. Pendimethaline is famous herbicide successfully applied nearly $82.5 \mathrm{~mL} \mathrm{ha}^{-1}$ to suppress cotton weeds as applied by Ali et al. (2019). Different growth, yield as well as fiber traits were examined. Agro-climatic conditions of experimental site CCRI were presented (Figure 1). 

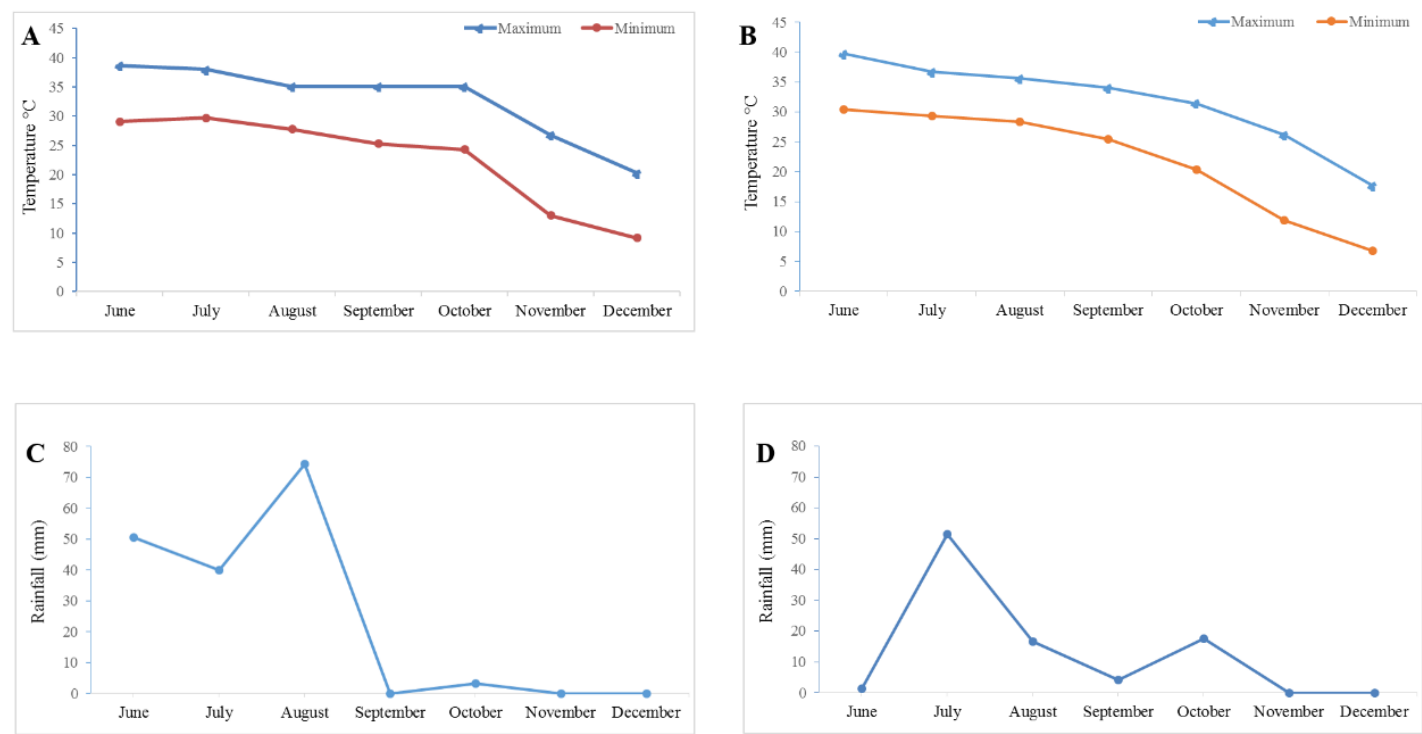

Figure 1. Eco-meteorological data of Multan, Punjab (Pakistan) during 2017 (A \& C) and during $2018(B \& D)$

\section{Yield related traits}

Plant height was determined through measuring scale. Average number of nodes and number of bolls/ plant were computed. Boll weight was measured through digital weighing balance. Lint yield, biological yield as well as harvest index were recorded as described (Apel and Hirt, 2004).

\section{Phenological related traits}

Leaf area index $(\mathrm{LAI})=$ Area of leaf/ ground leaf area

Crop growth rate was determined by dividing leaf area on dried leaf samples at $70{ }^{\circ} \mathrm{C}$ (Fang and Xiong, 2015). Lead area duration (LAD) was determined by measuring leaf surface area against time (Farooq et al., 2008). Net assimilation rate (NAR) was calculated by dividing relative growth rate on ratio of leaf area as previously mentioned (Fahad et al., 2016).

\section{Fiber related traits}

Cotton bolls were dried at room temperature and then their fiber was collected using ginning machine manually. All samples were subjected to $65 \%$ humidity at $20{ }^{\circ} \mathrm{C}$ for determination of fiber finess, fiber uniformity, fiber length, fiber elongation and fiber strength and micronairee were calculated according earlier adopted methods (Apel and Hirt, 2004; Fang and Xiong, 2015).

\section{Data analysis}

RCBD design was used to test the significance level of collected data through software Statistix 8.1. Moreover, means were separated through LSD test at 5\% probability level according to (Anjum et al., 2020). Economic analysis were performed by collecting input and output data, and benefit cost ration was determined by dividing net return on total cost as described (Ali et al., 2019). 


\section{Results}

\section{Yield related traits}

The cultivars, planting system and their interaction was significantly affected the yield traits in the present study except biological yield (Table 1). Regarding the individual effect of cultivars, Cultivar CIM-602 had significantly longer plant height $(163.56 \mathrm{~cm})$, while the cultivar CIM-678 had significantly smaller plant height $(157.67 \mathrm{~cm})$. Regarding the effect of planting systems, bed sowing had the largest plant height $(162.83 \mathrm{~cm})$ than flat sowing $(158.00 \mathrm{~cm})$. The interaction of cultivars as well as planting systems indicted that significantly largest plant height was recorded in cultivar CIM-602 $\times$ bed sowing $(165.33 \mathrm{~cm})$. The highest number of bolls/ plant (34.67) as well as boll weight $(1.90 \mathrm{~g})$ were estimated from cultivar CIM-602 than other studied cultivar. The interactive effect of cultivars as well as different planting systems described that the larger number of boll/ plant (36.33) as well as weight of boll $(2.11 \mathrm{~g})$ were measured from CIM-602 $\times$ bed sowing. Significantly the maximum seed cotton yield $\left(2246.6 \mathrm{Kg} \mathrm{ha}^{-1}\right)$ and lint yield (38.56 Kg ha-1) were found in cultivar CIM-602, however significantly meager seed cotton yield (2071.4 Kg ha-1) and lint yield (32.56 Kg ha-1) were calculated in cultivar CIM-678. Significantly greater seed cotton yield $\left(2278.2 \mathrm{Kg} \mathrm{ha}^{-1}\right)$ and lint yield $\left(37.67 \mathrm{Kg} \mathrm{ha}^{-1}\right)$ were determined in bed sowing than other planting systems. The significantly higher biological yield $\left(0.76 \mathrm{t} \mathrm{ha}^{-1}\right)$ and seed cotton harvest $(24.67 \%)$ were measured in cultivar CIM-602. Although, significantly meager biological yield $\left(0.71 \mathrm{t} \mathrm{ha}^{-1}\right)$ as well as seed cotton harvest $(19.44 \%)$ were found in CIM-678. Higher biological yield $\left(0.77 \mathrm{t} \mathrm{ha}^{-1}\right)$ and seed cotton harvest $(24.17 \%)$ were found on raised beds and lower biological yield $\left(0.65 \mathrm{t} \mathrm{ha}^{-1}\right)$ as well as seed cotton harvest $(19.50 \%)$ were present in flat beds. The interaction of cultivars as well as planting systems were depicted that the higher biological yield $\left(0.83 \mathrm{t} \mathrm{ha}^{-1}\right)$ and seed cotton harvest $(26.33 \%)$ were measured from CIM-602 $\times$ beds sowing (Table 2).

Table 1. Statistical analysis (LSD values) of different yield, physiological and fiber quality related traits of two cotton cultivars grown under different planting systems

\begin{tabular}{|c|c|c|c|}
\hline Trait & Cultivars & Planting systems & $\begin{array}{c}\text { Cultivars } \times \text { Planting } \\
\text { systems } \\
\end{array}$ \\
\hline Plant height $(\mathrm{cm})$ & $826.18 * *$ & $189.12 * *$ & $12.65 * *$ \\
\hline Number of bolls/plant & $240.00 * *$ & $67.81 * *$ & $8.44 * *$ \\
\hline Boll weight $(\mathrm{g})$ & $0.23 \mathrm{~ns}$ & $77.46^{* *}$ & $74.65 * *$ \\
\hline Seed cotton yield $\left(\mathrm{Kg} \mathrm{ha}^{-1}\right)$ & $108938 * *$ & $78231.4 * *$ & $3804.25 * *$ \\
\hline Lint yield $\left(\mathrm{Kg} \mathrm{ha}^{-1}\right)$ & $502.76 * *$ & $104.14 * *$ & $6.21 *$ \\
\hline Biological yield $\left(\mathrm{t} \mathrm{ha}^{-1}\right)$ & $9.88^{*}$ & $28.11^{* *}$ & $1.77 \mathrm{~ns}$ \\
\hline Seed cotton harvest $(\%)$ & $251.02 * *$ & $68.64 * *$ & $7.27 *$ \\
\hline Leaf area index & $130.39 * *$ & $44.66 * *$ & $8.97 * *$ \\
\hline Leaf area duration (days) & $2457.65 * *$ & $1060.41 * *$ & $2.19 \mathrm{~ns}$ \\
\hline Crop growth rate $\left(\mathrm{g} \mathrm{m}^{-2}\right.$ day $\left.^{-1}\right)$ & $1920.76 * *$ & $22.07 * *$ & $5.24 *$ \\
\hline Net assimilation rate $\left(\mathrm{g} \mathrm{m}^{-2}\right.$ day $\left.^{-1}\right)$ & $3082.92 * *$ & $37.07 * *$ & $7.76^{* *}$ \\
\hline Fiber finess & $3535.80 * *$ & $40.10 * *$ & $7.03 * *$ \\
\hline Fiber uniformity & $297.61 * *$ & $32.17 * *$ & $1.52 \mathrm{~ns}$ \\
\hline Fiber length (mm) & $846.40 * *$ & $69.10 * *$ & $4.30 *$ \\
\hline Fiber elongation (mm) & $1246.94 * *$ & $60.63 * *$ & $9.65 * *$ \\
\hline Fiber strength (mm) & $1209.14 * *$ & $91.86 * *$ & $6.14 *$ \\
\hline Micronaire $(\mu \mathrm{g} /$ inch $)$ & $1548.00 * *$ & $26.67 * *$ & $4.67 *$ \\
\hline
\end{tabular}

$\mathrm{ns}=$ non-significant, $*=$ significant at $\mathrm{p}=0.05$, and $* *=$ significant at $\mathrm{p}=0.01$ 
Table 2. Yield related traits of two cotton cultivars grown under different planting systems

\begin{tabular}{|c|c|c|c|c|c|}
\hline Traits & Cultivars & Flat sowing & Ridge sowing & Bed sowing & Mean \\
\hline \multirow{3}{*}{ Plant height $(\mathrm{cm})$} & CIM-678 & $154.33 \mathrm{f}$ & $158.33 \mathrm{e}$ & $160.33 \mathrm{~d}$ & $157.67 \mathrm{~b}$ \\
\hline & CIM-602 & $161.67 \mathrm{c}$ & $163.67 \mathrm{~b}$ & $165.33 \mathrm{a}$ & $163.56 \mathrm{a}$ \\
\hline & Mean & $158.00 \mathrm{c}$ & $161.00 \mathrm{~b}$ & $162.83 \mathrm{a}$ & \\
\hline \multirow{3}{*}{ Number of bolls/plant } & CIM-678 & $25.67 \mathrm{e}$ & $30.33 \mathrm{~d}$ & $32.00 \mathrm{c}$ & $29.33 \mathrm{~b}$ \\
\hline & CIM-602 & $33.00 \mathrm{c}$ & $34.67 \mathrm{~b}$ & $36.33 \mathrm{a}$ & $34.67 \mathrm{a}$ \\
\hline & Mean & $29.33 \mathrm{c}$ & $32.50 \mathrm{~b}$ & $34.16 \mathrm{a}$ & \\
\hline \multirow{3}{*}{ Boll weight (g) } & CIM-678 & $1.31 \mathrm{~d}$ & $1.70 \mathrm{bc}$ & $1.79 \mathrm{~b}$ & $1.60 \mathrm{~b}$ \\
\hline & CIM-602 & $1.59 \mathrm{c}$ & $1.99 \mathrm{a}$ & $2.11 \mathrm{a}$ & $1.90 \mathrm{a}$ \\
\hline & Mean & $1.45 \mathrm{c}$ & $1.45 \mathrm{c}$ & $1.45 \mathrm{c}$ & \\
\hline \multirow{3}{*}{$\begin{array}{l}\text { Seed cotton yield }(\mathrm{Kg} \\
\left.\mathrm{ha}^{-1}\right)\end{array}$} & CIM-678 & $1968.0 \mathrm{f}$ & $2072.3 \mathrm{e}$ & $2174.0 \mathrm{c}$ & $2071.4 \mathrm{~b}$ \\
\hline & CIM-602 & $2077.7 \mathrm{~d}$ & $2279.7 \mathrm{~b}$ & $2382.3 \mathrm{a}$ & $2246.6 \mathrm{a}$ \\
\hline & Mean & $2022.8 \mathrm{c}$ & $2176.0 \mathrm{~b}$ & $2278.2 \mathrm{a}$ & \\
\hline \multirow{3}{*}{ Lint yield $\left(\mathrm{Kg} \mathrm{ha}^{-1}\right)$} & CIM-678 & $29.33 \mathrm{f}$ & $33.33 \mathrm{e}$ & $35.00 \mathrm{~d}$ & $32.56 \mathrm{~b}$ \\
\hline & CIM-602 & $36.67 \mathrm{c}$ & $38.67 \mathrm{~b}$ & $40.33 \mathrm{a}$ & $38.56 \mathrm{a}$ \\
\hline & Mean & $33.00 \mathrm{c}$ & $36.00 \mathrm{~b}$ & $37.67 \mathrm{a}$ & \\
\hline \multirow{3}{*}{ Biological yield $\left(\mathrm{t} \mathrm{ha}^{-1}\right)$} & CIM-678 & $0.65 \mathrm{c}$ & $0.74 \mathrm{~b}$ & $0.75 \mathrm{~b}$ & $0.71 \mathrm{~b}$ \\
\hline & CIM-602 & $0.66 \mathrm{c}$ & $0.80 \mathrm{ab}$ & $0.83 \mathrm{a}$ & $0.76 \mathrm{a}$ \\
\hline & Mean & $0.65 \mathrm{~b}$ & $0.77 \mathrm{a}$ & $0.79 \mathrm{a}$ & \\
\hline \multirow{3}{*}{ Seed cotton harvest $(\%)$} & CIM-678 & $16.00 \mathrm{e}$ & $20.33 \mathrm{~d}$ & $22.00 \mathrm{c}$ & $19.44 \mathrm{~b}$ \\
\hline & CIM-602 & $23.00 \mathrm{c}$ & $24.67 \mathrm{~b}$ & $26.33 \mathrm{a}$ & $24.67 \mathrm{a}$ \\
\hline & Mean & $19.50 \mathrm{c}$ & $22.50 \mathrm{~b}$ & $24.17 \mathrm{a}$ & \\
\hline
\end{tabular}

* Mean values sharing similar letter(s) in a column are statistically non-significant at $\mathrm{p}=0.05$ (LSD test)

\section{Phenological related traits}

The cultivars and planting system and their combined effect were significantly affected the phenological traits (Table 1). The significantly greater leaf area index (0.44) as well as leaf area duration (544.44 days) were calculated from cultivar CIM-602, while significantly lower leaf area index (0.38) as well as leaf area duration (427.78 days) were estimated from CIM-678. The maximum leaf area duration (536.83 days) and leaf area index (0.44) were calculated from beds sown cotton, while lower leaf area duration (411.00 days) and leaf area index (0.38) were calculated from flats sown plants. The interactive effects of two cultivars under three different planting systems showed that the much leaf area duration (592.00 days) and leaf area index $\left(0.48 \mathrm{t} \mathrm{ha}^{-1}\right)$ were measured in CIM-602 $\times$ beds than all other studied combinations. Significantly greater crop growth rate $\left(5.07 \mathrm{~g} \mathrm{~m}^{-2}\right.$ day $\left.^{-1}\right)$ and net assimilation rate $\left(1.35 \mathrm{~g} \mathrm{~m}^{-2} \mathrm{day}^{-1}\right)$ were calculated cultivar CIM-602, while significantly lower crop growth rate $\left(4.79 \mathrm{~g} \mathrm{~m}^{-2}\right.$ day $\left.^{-1}\right)$ and net assimilation (1.01 $\left.\mathrm{g} \mathrm{m}^{-2} \mathrm{day}^{-1}\right)$ were calculated cultivar CIM-678. The maximum crop growth rate $\left(4.95 \mathrm{~g} \mathrm{~m}^{-2} \mathrm{day}^{-1}\right)$ and net assimilation rate $\left(1.21 \mathrm{~g} \mathrm{~m}^{-2}\right.$ day $\left.^{-1}\right)$ were calculated from bed sowing, while lower crop growth rate $\left(4.90 \mathrm{~g} \mathrm{~m}^{-2} \mathrm{day}^{-1}\right)$ and net assimilation rate $\left(1.14 \mathrm{~g} \mathrm{~m}^{-2} \mathrm{day}^{-1}\right)$ were calculated from flat sowing. The interactive effects of cultivars under diverse planting systems showed that the higher crop growth rate 
$\left(5.10 \mathrm{~g} \mathrm{~m}^{-2}\right.$ day $\left.^{-1}\right)$ and net assimilation rate $\left(1.38 \mathrm{~g} \mathrm{~m}^{-2}\right.$ day $\left.^{-1}\right)$ were measured in CIM-602 $\times$ bed sowing than all other studied combinations (Table 3 ).

Table 3. Physiological related traits of two cotton cultivars grown under different planting systems

\begin{tabular}{c|c|c|c|c|c}
\hline Traits & Cultivars & Flat sowing & Ridge sowing & Bed sowing & Mean \\
\hline \multirow{4}{*}{ Leaf area index } & CIM-678 & $0.36 \mathrm{~d}$ & $0.39 \mathrm{c}$ & $0.40 \mathrm{c}$ & $0.38 \mathrm{~b}$ \\
& CIM-602 & $0.39 \mathrm{c}$ & $0.46 \mathrm{~b}$ & $0.48 \mathrm{a}$ & $0.44 \mathrm{a}$ \\
& Mean & $0.38 \mathrm{c}$ & $0.42 \mathrm{~b}$ & $0.44 \mathrm{a}$ & \\
\hline \multirow{2}{*}{ Leaf area duration } & CIM-678 & $352.33 \mathrm{f}$ & $449.33 \mathrm{e}$ & $481.67 \mathrm{c}$ & $427.78 \mathrm{~b}$ \\
(days) & CIM-602 & $469.67 \mathrm{~d}$ & $571.67 \mathrm{~b}$ & $592.00 \mathrm{a}$ & $544.44 \mathrm{a}$ \\
& Mean & $411.00 \mathrm{c}$ & $510.50 \mathrm{~b}$ & $536.83 \mathrm{a}$ & \\
\hline \multirow{2}{*}{ Crop growth rate $_{\left(\mathrm{g} \mathrm{m}^{-2} \text { day }^{-1}\right)}$} & CIM-678 & $4.78 \mathrm{~d}$ & $4.80 \mathrm{~cd}$ & $4.81 \mathrm{c}$ & $4.79 \mathrm{~b}$ \\
& CIM-602 & $5.03 \mathrm{~b}$ & $5.09 \mathrm{a}$ & $5.10 \mathrm{a}$ & $5.07 \mathrm{a}$ \\
\hline \multirow{2}{*}{ Net assimilation rate } & Mean & $4.90 \mathrm{~b}$ & $4.94 \mathrm{a}$ & $4.95 \mathrm{a}$ & \\
$\left(\mathrm{g} \mathrm{m}^{-2}\right.$ day $\left.^{-1}\right)$ & CIM-678 & $0.99 \mathrm{e}$ & $1.02 \mathrm{~d}$ & $1.03 \mathrm{~d}$ & $1.01 \mathrm{~b}$ \\
& Mean & $1.29 \mathrm{c}$ & $1.36 \mathrm{~b}$ & $1.38 \mathrm{a}$ & $1.35 \mathrm{a}$ \\
\hline
\end{tabular}

* Mean values sharing similar letter(s) in a column are statistically non-significant at $\mathrm{p}=0.05$ (LSD test)

\section{Fiber related traits}

The cultivars and planting system and their combined effect were significantly affected the fiber quality related traits (Table 1). Cultivar CIM-602 had the highest fiber finess (3.44) and fiber uniformity (47.89), while cultivar CIM-602 had the lowest fiber finess (3.09) and fiber uniformity (39.67). Significantly greater fiber finess (3.29) and uniformity (46.00) were collected from beds sown plants, however significantly meager fiber finess (3.23) and uniformity (41.33) were recorded from flat sowing. The greater fiber length $(33.67 \mathrm{~mm})$ and elongation $(30.21 \mathrm{~mm})$ were recorded from cultivar CIM-602, while the lower fiber length $(23.44 \mathrm{~mm})$ and elongation $(30.06 \mathrm{~mm})$ were measured in CIM-678. The maximum fiber length $(30.83 \mathrm{~mm})$ and elongation $(30.16 \mathrm{~mm})$ were shown in bed plantings. The minimum fiber length $(25.83 \mathrm{~mm})$ and elongation $(30.11 \mathrm{~mm})$ were measured from flat plantings. The significantly higher fiber strength $(25.56 \mathrm{~mm})$ and micronairee $(4.17 \mu \mathrm{g} / \mathrm{inch})$ were recorded from CIM-602 cultivar, while the significantly lower fiber strength $(15.33 \mathrm{~mm})$ and micronairee $(3.83 \mu \mathrm{g} / \mathrm{inch})$ were measured in cultivar CIM-678. The greater fiber strength $(22.67 \mathrm{~mm})$ and micronairee (4.05 $\mu \mathrm{g} /$ inch) were assessed from beds sown plants, while the lower fiber strength $(17.83 \mathrm{~mm})$ as well as micronaire $(3.99 \mu \mathrm{g} / \mathrm{inch})$ were measured in those plants harvested from flat beds. The interactive effect of cultivars under planting systems mentioned that highest fiber strength $\left(27.33 \mathrm{~g} \mathrm{~m}^{-2}\right.$ day $\left.^{-1}\right)$ as well as micronaire $(4.21 \mu \mathrm{g} /$ inch $)$ were measured from CIM-602 $\times$ beds (Table 4).

Benefit cost ratio was higher on bed sowing and lower was recorded from flat sowing in both years study. The benefit ratio was higher on bed sowing cultivation. Detailed description of economic analysis was presented (Table 5). 
Table 4. Fiber quality related traits of two cotton cultivars grown under different planting systems

\begin{tabular}{|c|c|c|c|c|c|}
\hline Traits & Cultivars & Flat sowing & Ridge sowing & Bed sowing & Mean \\
\hline \multirow{3}{*}{ Fiber finess } & CIM-678 & $3.07 \mathrm{e}$ & $3.10 \mathrm{~d}$ & $3.11 \mathrm{~d}$ & $3.09 \mathrm{~b}$ \\
\hline & CIM-602 & $3.39 \mathrm{c}$ & $3.46 \mathrm{~b}$ & $3.48 \mathrm{a}$ & $3.44 \mathrm{a}$ \\
\hline & Mean & $3.23 \mathrm{c}$ & $3.28 \mathrm{~b}$ & $3.29 \mathrm{a}$ & \\
\hline \multirow{3}{*}{ Fiber uniformity } & CIM-678 & $36.67 \mathrm{~d}$ & $40.33 \mathrm{c}$ & $42.00 \mathrm{c}$ & $39.67 \mathrm{~b}$ \\
\hline & CIM-602 & $46.00 \mathrm{~b}$ & $47.67 \mathrm{~b}$ & $50.00 \mathrm{a}$ & $47.89 \mathrm{a}$ \\
\hline & Mean & $41.33 \mathrm{c}$ & $44.00 \mathrm{~b}$ & $46.00 \mathrm{a}$ & \\
\hline \multirow{3}{*}{ Fiber length (mm) } & CIM-678 & $20.00 \mathrm{f}$ & $24.33 \mathrm{e}$ & $26.00 \mathrm{~d}$ & $23.44 \mathrm{~b}$ \\
\hline & CIM-602 & $31.67 \mathrm{c}$ & $33.67 \mathrm{~b}$ & $35.67 \mathrm{a}$ & $33.67 \mathrm{a}$ \\
\hline & Mean & $25.83 \mathrm{c}$ & $29.00 \mathrm{~b}$ & $30.83 \mathrm{a}$ & \\
\hline \multirow{3}{*}{ Fiber elongation (mm) } & CIM-678 & $30.04 \mathrm{e}$ & $30.07 \mathrm{~d}$ & $30.08 \mathrm{~d}$ & $30.06 \mathrm{~b}$ \\
\hline & CIM-602 & $30.17 \mathrm{c}$ & $30.22 \mathrm{~b}$ & $30.24 \mathrm{a}$ & $30.21 \mathrm{a}$ \\
\hline & Mean & $30.11 \mathrm{c}$ & $30.15 \mathrm{~b}$ & $30.16 \mathrm{a}$ & \\
\hline \multirow{3}{*}{ Fiber strength (mm) } & CIM-678 & $12.00 \mathrm{f}$ & $16.00 \mathrm{e}$ & $18.00 \mathrm{~d}$ & $15.33 b$ \\
\hline & CIM-602 & $23.67 \mathrm{c}$ & $25.67 \mathrm{~b}$ & $27.33 \mathrm{a}$ & $25.56 \mathrm{a}$ \\
\hline & Mean & $17.83 \mathrm{c}$ & $20.83 \mathrm{~b}$ & $22.67 \mathrm{a}$ & \\
\hline \multirow{3}{*}{ Micronaire ( $\mu \mathrm{g} /$ inch $)$} & CIM-678 & $3.86 \mathrm{~d}$ & $3.89 \mathrm{~cd}$ & $3.90 \mathrm{c}$ & $3.88 \mathrm{~b}$ \\
\hline & CIM-602 & $4.12 \mathrm{~b}$ & $4.18 \mathrm{a}$ & $4.21 \mathrm{a}$ & $4.17 \mathrm{a}$ \\
\hline & Mean & $3.99 \mathrm{~b}$ & $4.03 \mathrm{a}$ & $4.05 \mathrm{a}$ & \\
\hline
\end{tabular}

Mean values sharing similar letter(s) in a column are statistically non-significant at $\mathrm{p}=0.05$ (LSD test)

Table 5. Role of planting systems on benefit cost ratio (BCR) of cotton

\begin{tabular}{|c|c|c|c|c|c|c|c|c|}
\hline & Treatments & $\underset{\left(\mathrm{kg} \mathrm{ha}^{-1}\right)}{\text { Yield }}$ & $\begin{array}{l}\text { Value } \\
\left(\$ \text { ha }^{-1}\right)\end{array}$ & $\begin{array}{c}\text { stick value } \\
\left(\$ \text { ha }^{-1}\right)\end{array}$ & $\begin{array}{l}\text { Gross } \\
\text { value } \\
\left(\$ \text { ha }^{-1}\right)\end{array}$ & $\begin{array}{c}\text { Total cost } \\
\left(\$ \mathbf{h a}^{-1}\right)\end{array}$ & $\begin{array}{c}\text { Net return } \\
\left(\$ \mathrm{ha}^{-1}\right)\end{array}$ & BCR \\
\hline \multirow{3}{*}{2017} & Flat sowing & 2500 & 1170.866 & 7.751938 & 1178.618 & 723.1912 & 455.4264 & 1.63 \\
\hline & Ridge sowing & 2630 & 1231.751 & 7.751938 & 1239.503 & 733.2041 & 506.2984 & 1.69 \\
\hline & Bed sowing & 2880 & 1348.837 & 7.751938 & 1356.589 & 715.4393 & 641.1499 & 1.90 \\
\hline \multirow{3}{*}{2018} & Flat sowing & 2550 & 1194.283 & 7.751938 & 1202.035 & 723.4496 & 478.5853 & 1.66 \\
\hline & Ridge sowing & 2680 & 1255.168 & 7.751938 & 1262.92 & 733.2687 & 529.6512 & 1.72 \\
\hline & Bed sowing & 2930 & 1372.255 & 7.751938 & 1380.006 & 715.5685 & 664.438 & 1.93 \\
\hline
\end{tabular}

Note: Cotton price $=16.15 \$ / 40 \mathrm{~kg}($ during 2017) and $18.73 \$ / 40 \mathrm{~kg}$ (during 2018)

\section{Discussion}

Regarding the mean performance of cultivars, cultivar CIM-602 showed the highest growth related traits i.e. plant height, number of bolls/ plant, boll weight, seed cotton yield, lint yield, biological yield and seed cotton harvest than other cultivar CIM-678. Regarding the effect of three different planting systems i.e. flats, ridges and beds, the excellent increase was exhibited in yield related traits of cotton cultivars from bed cultivation among other studied planting systems. Furthermore, interactive effect of cultivars as well as planting systems were potentially examined the involvement of planting systems in cotton production. The yield related traits were also increased under 
interactive effect of cultivar CIM-602 and bed sowing. Current study is under conformity of earlier research who reported that bed sowing increased the yield of different agronomic crops especially cotton crop (Ali et al., 2016). Similarly, Duzy and Kornecki (2019) evaluated that suitable sowing method increased the cotton yield. In earlier decades, the ridge sowing is found to satisfactory for farmers as reported (Anjum et al., 2019). However, under water stress conditions, beds sowing is excellent and preferable planting system for best cotton productivity and ultimately higher yield can successfully be accomplished.

Regarding the physiological traits, the greater leaf area index, leaf area duration, crop growth rate and net assimilation rate were recorded in cultivar CIM-602 than other one cultivar CIM-678. Concerning the effect of planting systems, bed plantings of cotton cultivars showed the higher net assimilation rate, crop growth rate, leaf area duration and leaf area index than other two different planting methods. However, poor performance among physiological traits were measured from flat sowing. Furthermore, combined effect of cultivars like CIM-678 and CIM-602 and three planting methods such as flats, ridges and beds planting exhibited a greater involvement in improvement of cotton production. The net assimilation rate, crop growth rate, leaf area duration and leaf area index were increased from cultivar CIM-602 and bed plantings than other one cultivar CIM-678 and flat plantings. Current study indicated that appropriate use of tillage system had excellent capability to increase the production of agronomic crops especially cotton cultivars. In the current study, bed sowing is best tillage system for higher physiological related traits, because these physiological traits were involved in increase of growth and development of cotton crop (Nadeem et al., 2013). The highest physiological traits were attained from CIM-602 and bed sowing than other planting system. It has been assumed that water stress condition is very critical constrain that involved in reduction of plant growth and yield as in earlier work of Mahpara et al. (2019). Moreover, different suitable cultural practices, nutritional balance and selection of cultivars were played an imperative role in higher crop productivity of cotton crop with superior quality of cotton.

Fiber excellence related parameters such as fiber finess, uniformity, elongation, strength and micronaire were found to be effective for farmers and further for industrial purposes. Concerning the cultivars, the higher fiber related parameters like fiber finess, uniformity, elongation, strength as well as micronaire were measured from cultivar CIM-602 as compared to cultivar CIM-678. Regarding the tillage system, bed sowing provides the highest fiber finess, uniformity, elongation, strength as well as micronaire than other considered tillage methods. The highest moisture content was available to plants in beds plantings. Regarding the interactive consequence of cultivars as well as planting system, significantly greater fiber traits were found in cultivar CIM-602 as well as bed planting system. Hence, current study is more effective for attaining appropriate fiber traits. Current study indicated the benefits of beds sowing on fiber related traits (Zohaib et al., 2018). Several previous studies confirmed that bed sowing improved the fiber related attributes such as fiber finess, elongation, uniformity, strength and micronaire as recorded in current findings (Shah et al., 2011; Upadhyaya and Panda, 2019). Correspondingly, Ali et al. (2020) also determined that improvement fiber quality is more important for farmers and industry and use of appropriate cultivars is much imperative. Hence, current is helpful and encourages the use of suitable tillage system for cotton production with good quality fiber traits. The increase of net return and higher benefit cost ratio is found to be effective for increase of production and their earnings. 


\section{Conclusion}

Cotton is a major oil-producing crop, but the effects of the cultivation on the oil production had not been studied. It has been concluded that CIM-602 is important cultivar and performed better under water stress conditions than CIM-678. Regarding the tillage system, bed sowing is important for higher production than flat and ridge sowing. CIM-602 and ridge swing are favorable for farmers and required very low inputs. It has been recommended that the cultivation of CIM-602 on bed sowing increases the farmer's earnings and providing higher benefit cost ratio.

Acknowledgements. The authors are highly grateful to the Bahauddin Zakariya University, Multan for financial support to conduct the study.

\section{REFERENCES}

[1] Ahmad, R., Malik, W., Anjum, M. A. (2019): Genetic diversity and selection of suitable molecular markers for characterization of Indigenous Zizyphus germplasm. - ErwerbsObstbau 61(4): 345-353.

[2] Ali, M., Ali, L., Sattar, M., Ali, M. A. (2010): Response of seed cotton yield to various plant populations and planting methods. - J. Agric. Res. 48(2): 163-169.

[3] Ali, H., Tariq, N., Ahmad, S., Chattha, T. H., Hussain, A. (2012): Effect of irrigation at different growth stages and phosphorus application methods on agronomic traits of wheat (Triticum aestivum L.). - J. Food Agric. Environ. 10: 1371-1375.

[4] Ali, F., Ali, A., Gul, H., Sharif, M., Sadiq, A., Ahmed, A., Ullah, A., Mahar, A., Kalhoro, S. A. (2015): Effect of boron soil application on nutrients efficiency in tobacco leaf. Amer. J. Plant Sci. 6(09): 1391.

[5] Ali, M., Ali, L., Waqar, M. Q., Ali, M. A. (2016): Bed planting: a new crop establishment method for wheat (Triticum aestivum L.) in cotton-wheat cropping system of southern Punjab. - Int. J. Agric. Appl. Sci. 47: 777-780.

[6] Ali, H., Ahmad, A., Hussain, S. (2019): The effect of exogenous phosphorous application on growth, yield, quality and net returns of Upland cotton (Gossipium hirsutum L.). Applied Ecology and Environmental Research 18(1): 769-781.

[7] Ali, H., Ahmad, A. and Hussain, S. (2020): The effect of exogenous phosphorous application on growth, yield, quality and net returns of upland cotton (Gossipium hirsutum L.). - Appl. Ecol. Env. Res. 18(1): 769-781.

[8] Amouzou, K. A., Naab, J. B., Lamers, J. P., Borgemeister, C., Becker, M., Vlek, P. L. (2018): CROPGRO-Cotton model for determining climate change impacts on yield, waterand N-use efficiencies of cotton in the Dry Savanna of West Africa. - Agric. Systems 165: 85-96.

[9] Anjum, M. A., Muhammad, H. M. D., Balal, R. M., Ahmad, R. (2019): Performance of two onion (Allium cepa L.) cultivars under two different planting systems in calcareous soil. - J. Hortic. Sci. Technol. 2(2): 54-59.

[10] Anjum, M. A., Haram, A., Ahmad, R., Bashir, M. A. (2020): Physico-chemical attributes of fresh and dried Indian jujube (Zizyphus mauritiana) fruits. - Pak. J. Agric. Sci. 57(1): 165-176.

[11] Apel, K., Hirt, H. (2004): Reactive oxygen species: metabolism, oxidative stress, and signal transduction. - Ann. Review Plant Biol 55: 373-399.

[12] Bellaloui, N., Turley, R. B., Stetina, S. R. (2015): Water stress and foliar boron application altered cell wall boron and seed nutrition in near-isogenic cotton lines expressing fuzzy and fuzzless seed phenotypes. - PloS one 10(6): e0130759. 10.1371/journal.pone.0130759. 
[13] Duzy, L. M., Kornecki, T. S. (2019): Effects of cover crop termination and cotton planting methods on cotton production in conservation systems. - Renew. Agric. Food Syst. 34(5): 406-414.

[14] Fahad, S., Hussain, S., Saud, S., Khan, F., Hassan, S., Nasim, W., Arif, M., Wang, F., Huang, J. (2016): Exogenously applied plant growth regulators affect heat-stressed rice pollens. - J. Agron. Crop Sci. 202(2): 139-150.

[15] Fang, Y., Xiong, L. (2015): General mechanisms of drought response and their application in drought resistance improvement in plants. - Cell. Mol. Life Sci. 72: 673-689.

[16] Farooq, M., Basra, S. M. A., Wahid, A., Cheema, Z. A., Cheema, M. A., Khaliq, A. (2008): Physiological role of exogenously applied glycinebetaine in improving drought tolerance of fine grain aromatic rice (Oryza sativa L.). - J. Agron. Crop Sci. 194: 325-333.

[17] Magare, P. N., Jadhao, S. D., Farkade, B. K., Mali, D. V. (2018): Effect of levels of potassium on yield, nutrient uptake, fertility status and economics of cotton grown in Vertisol. - Int. J. Curr. Microbiol. Appl. Sci. 7(4): 1292-1300.

[18] Mahpara, S., Shahnawaz, M., Rehman, K., Ahmad, R., Khan, F. U. (2019): Nitrogen fertilization induced drought tolerance in sunflower: a review. - Pure App. Biol. 8(2): 16751683.

[19] Nadeem, M. A., Idrees, M., Ayub, M., Tanveer, A., Mubeen, K. (2013): Effect of different weed control practices and planting systems on weeds and yield of cotton. - Pak. J. Bot. 45(4): 1321-1328.

[20] Pettigrew, W. T., Dowd, M. K. (2011): Varying planting dates or irrigation regimes alters cottonseed composition. - Crop Sci. 51(5): 2155-2164.

[21] Rad, N. M. R., Kadir, M. A., Yusop, M. R. (2012): Genetic behaviour for plant capacity to produce chlorophyll in wheat (Triticum aestivum) under drought stress. - Aust. J. Crop Sci. 6(3): 415.

[22] Shah, A. R., Khan, T. M., Sadaqat, H. A., Chatha, A. A. (2011): Alterations in leaf pigments in cotton (Gossypium hirsutum) genotypes subjected to drought stress conditions. - Int. J. Agric. Biol. 13(6): 902-908.

[23] Upadhyaya, H., Panda, S. K. (2019): Drought stress responses and its management in rice. - In: Advances in Rice Research for Abiotic Stress Tolerance. Woodhead Publishing, pp. 177-200.

[24] Zohaib, A., Jabbar, A., Ahmad, R., Basra, S. M. A. (2018): Comparative productivity and seed nutrition of cotton by plant growth regulation under deficient and adequate boron conditions. - Planta Daninha 36: 12-17. 